\title{
Fat embolism syndrome following caesarean section in an obese patient and its histopathological similarity to an animal model of FE: a case report
}

\author{
Ogugua Ajemba ${ }^{1}$, Hamid Zia ${ }^{1}$, Kamani Lankachandra ${ }^{1}$, Gurmukh Singh ${ }^{1}$, Alan Poisner ${ }^{2}$, Betty \\ Herndon $^{3}$, Agostino Molteni ${ }^{1}$
}

1. Department of Pathology, University of Missouri at Kansas City, School of Medicine and Truman Medical Center, Kansas City, MO, United States. 2. Department of Pharmacology, University of Kansas Medical Center, Kansas City, Kansas, United States. 3. Departments of Medicine and Basic Medical Science, University of Missouri at Kansas City School of Medicine, United States.

Correspondence: Agostino Molteni. Address: Department of Pathology, University of Missouri at Kansas City, School of Medicine, 2411 Holmes Street, Kansas City, MO, United States. E-mail: moltenia@umkc.edu

Received: January 9, 2015

DOI : $10.5430 /$ crcp.v2n3p30
Accepted: February 26, 2015

URL: http://dx.doi.org/10.5430/crcp.v2n3p30

\section{Abstract}

We present the case of a 29 year-old primigravid obese female with a complex medical history, who underwent caesarean section at 35 weeks gestation due to preeclampsia. She has a past medical history of bilateral foot surgery, and bilateral hip pinning for chronic hip pain in 2012. Postoperatively, approximately six hours after surgery, she was in respiratory distress and 47 hours later she was found dead. At autopsy, a diagnosis of pulmonary fat embolism was made. Pulmonary histopathological examination revealed findings similar to an animal model of fat embolism.

\section{Key words}

Lungs, Fat embolism, Pulmonary hypertension, Pulmonary fibrosis

\section{I ntroduction}

In humans, fat embolism (FE) secondary to long bone fractures, liposuction, surgical procedures on the vertebral column, or to other traumas may lead to either a negligible lung injury or, much less frequently, to a clinically serious acute respiratory distress syndrome (ARDS) ${ }^{[1-3]}$.

In an early phase, some patients may experience acute respiratory insufficiency, petechial hemorrhages and mental disturbances; however, unless death occurs as consequence of fat embolism syndrome (FES) and ARDS, little or no clinical evidence of emboli persists. There is no information about the histopathological damage to the lungs of patients who suffered from FES, after the initial event, but recently it was reported in follow-up studies on the 2 and 5 year clinical outcome of patients with ARDS, FES included, that none of them returned to the normally expected predicted level of their physical respiratory function ${ }^{[4,5]}$. Similarly, there is a dearth of histopathological studies in experimental animals undergoing fat embolism. Most FE models have been limited to short term studies (hours, or a few days) and used "fat" such as fatty acid or corn oil not comparable to the fat of the bone marrow ${ }^{[6,7]}$. The only long term study using the most 
prominent fat of the bone marrow, the neutral fat triolein, was performed in dogs, by giving a 30 minute injection and it did not show any pulmonary fat accumulation seven days post the injection with a normal histological appearance of the organ within four weeks ${ }^{[8]}$. Such a rapid clearance and resolution could be the consequence of the prolonged time of the injection widely different from what happens following acute trauma.

In our recent study using i.v. injections of triolein in the rat caudal vein, we found that the pulmonary response to the FE induced by the triolein included not only a severe, acute, pulmonary vasoconstriction and consequent dilatation of the right heart ventricle, but also the occurrence of a rapid development of a lung fibrosis both in the small caliber artery wall and adventitia and in the pulmonary septa. This was associated with an increased presence of neutrophils, macrophages, plasma cells, mast cells and fibroblasts, the latter cells prominently expressing smooth muscle actin 1 (SMA 1) ${ }^{[9]}$.

Trichrome stains showed diffuse fibrosis ${ }^{[10]}$. Preliminary data from additional studies indicate that, after a period of recovery from this pulmonary damage (within 11 days), the chronic inflammatory and vascular process persists up to 6-10 weeks ${ }^{[11]}$, with fat droplets still present at the time. The Process is aggravated severely by a "second hit" done by i.p. injection of lipopolysaccharide (LPS) which per se only caused modest inflammatory lung damage ${ }^{[11,12]}$. The histopathological pulmonary damage of this experimental FES shows striking similarities with the changes observed at the autopsy examination in the lungs of our case report.

\section{Case presentation}

Patient is a 29-yr-old overweight primigravida who was admitted to the hospital at 36-week gestation for management of preeclampsia. She had a complicated medical history including spina bifida, status post correction of a myelomeningocele, complicated by neurogenic bowel and urinary bladder and chronic kidney disease. She had multiple surgical procedures including spinal, hip and foot surgeries two years prior to this admission. She received Albuterol for her asthma and home oxygen for obstructive sleep apnea. Pulmonary function tests performed four months prior demonstrated reduced FEV1 and FVC with normal FEV1/FVC ratio.

Physical examination revealed a 29-yr-old Caucasian female. Her vital signs were the following: temperature 97.1, blood pressure 154/101 mmHg, pulse 124 beats per minute, and respiration 18 beats per minute. Her weight was 169 pounds (76.8 kg) and her height was 61 inches $(134 \mathrm{~cm})$. Her lungs were clear to auscultation bilaterally and her heart rate was normal. No murmurs found. Her abdomen was soft without tenderness.

During her hospital stay, due to worsening severe preeclampsia, she underwent primary classical cesarean section under general anesthesia, at 38-week gestation after failed labor induction. A vertical midline skin incision was made just above and around the umbilicus to the right and down the mid portion of the lower abdomen to approximately $4 \mathrm{~cm}$ below the umbilicus. The abdominal fat was manipulated and the incision was taken down through the subcutaneous adipose tissue. Fetus was delivered. There was some postpartum hemorrhage but the rest of the procedure and delivery of the baby were uneventful. After surgery, the patient was transferred to the intensive care unit due to acute respiratory failure that improved on second post-operative day and was successfully extubated. On third post-operative day at approximately 53 hours following surgery, she was found to be in asystole. Resuscitation efforts were unsuccessful.

\subsection{Autopsy report}

An autopsy was performed to ascertain the cause of death. Sections of bilateral lungs showed evidence of acute pulmonary edema and multiple foreign body granulomas. Some of the blood vessels exhibited optical clearing of the lumens suggestive of fat emboli.

On histopathological examination, small caliber arteries and arterioles had increased media thickness with reduced lumen patency, and diffuse pneumonia with neutrophils, macrophages, lymphocytes and plasma cells present in the adventitia of 
vessels, the septa and the alveolar lumen. Bronchial inflammation was alternated with areas of de-epithelization (see Figure 1a). Oil Red O stain demonstrated numerous conspicuously scattered fat droplets in alveolar spaces and distal, smaller blood vessels in different areas of the lung (see Figure 1b). Immunohistochemical stain for pancytokeratin and special stain for mucin were performed on lung sections and were negative for fetal squamous or mucinous debris. Trichrome stain showed early interstitial fibrosis and thickening of the adventitia of the small and medium caliber pulmonary arteries and arterioles, in both the peribronchial musculature and the lobular septae (see Figure 1c). Smooth muscle actin (SMA) also showed increased staining in the media of medium sized blood Vessels (see Figure 1d). Neither upper body rash nor fat globules in bone marrow were noted. Based on these findings, the immediate cause of death was determined to be pulmonary fat embolism. Fat emboli are known to be present 24-72 hours after the initial insult with the peak incidence at 48 hours ${ }^{[1,2]}$. The origin of the fat emboli in this case was thought to be most likely related to mechanical abdominal adipose tissue disruption. Amniotic fluid embolism was excluded based on pulmonary histopathology and clinical timeline.

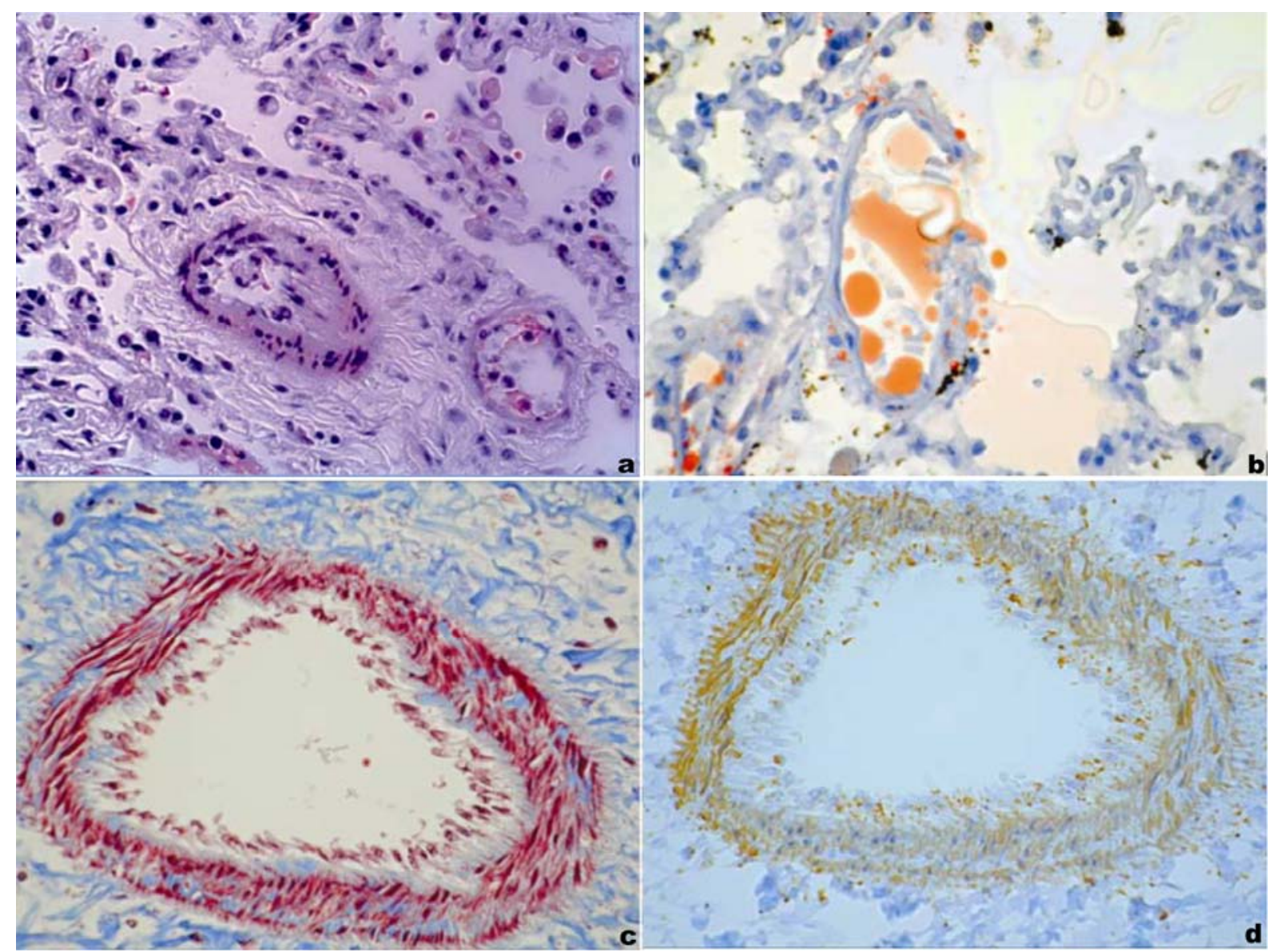

Figure 1. Lung of the patient. (a) H\&E staining shows small caliber arteries and arterioles with increased media thickness and reduction of lumen, inflammatory cells are present in the alveoli and the septal wall with areas of de-epithelization ( $\times 400)$; (b) Oil Red O stain shows numerous conspicuously scattered fat droplets in alveolar spaces of the lung $(\times 400)$; (c) Trichrome stain shows interstitial fibrosis and thickening of the adventitia of the arteries walls $(\times 400)$; (d) Smooth muscle actin (SMA) shows increased staining in the media of medium caliber blood vessels $(\times 400)$.

\subsection{Description of parallel histopathological findings in the rat model ( rats tested with triolein)}

As we reported in our article for Journal of Orthopedic Research ${ }^{[9]}$ we used 44 Sprague-Dawley rats (280 g - $300 \mathrm{~g}$ ) supplied by Harlan Laboratories, Indianapolis, IN. The University of Kansas animal care Committee approved the 
protocol. A dose of $0.2 \mathrm{ml}$ of pure glyceryl trioleate, (Sigma-Aldrich Corp, St. Louis, MO) was injected in the caudal vein of 31 unanaesthetized rats while 13 controls received in same vein $0.2 \mathrm{ml}$ of saline. The dose was selected from results in previous experiments. Food and water were given adlibitum and rats weight and behavior checked periodically. A few triolein injected animals died within 8 hours at different interval times before the planned euthanasia. The surviving rats were sacrificed by i.p. injections of Beuthanasia (Shering-Plough Animal Health Corp, Union, NJ) at $24 \mathrm{~h}$ ( $\mathrm{n}=6$ triolein, 5 control), $48 \mathrm{~h}$ ( $\mathrm{n}=5$ triolein, 4 control), $96 \mathrm{~h}$ ( $\mathrm{n}=5$ triolein), and 11 days ( $\mathrm{n}=5$ triolein, 4 controls). Body weight was taken before sacrifice.

After sacrifice lungs were weighed, placed with exception of a lobe in $10 \%$ buffererd formalin and after fixation paraffin embedded for histological studies. The portion of the lungs not fixed was frozen and Red Oil O stained. The formalin fixed portion of the organs were stained for H\&E, Masson Trichrome (for collagen) and alpha-smooth actin (SMA) (for fibrosis).

On histopathology examination, lungs showed severe vaso-constriction and thickening of the media of the same vessels and with decreased lumen patency, diffuse presence of neutrophils, macrophages, plasma cells and occasional mast cells, with peak of damage severity at 48-hours (see Figure 2a). Oil Red O stain showed fat droplets were evident in the lumen of small caliber arteries and arterioles in the lungs of rats sacrificed at the all interval times but with a peak at 48 hours (see Figure 2b). Trichrome and SMA stains showed, (see Figure 2c and 2d) fibrosis in the same vessels and in the septa (for Trichrome and SMA stain sections of rat lungs were also taken at 48 hours).

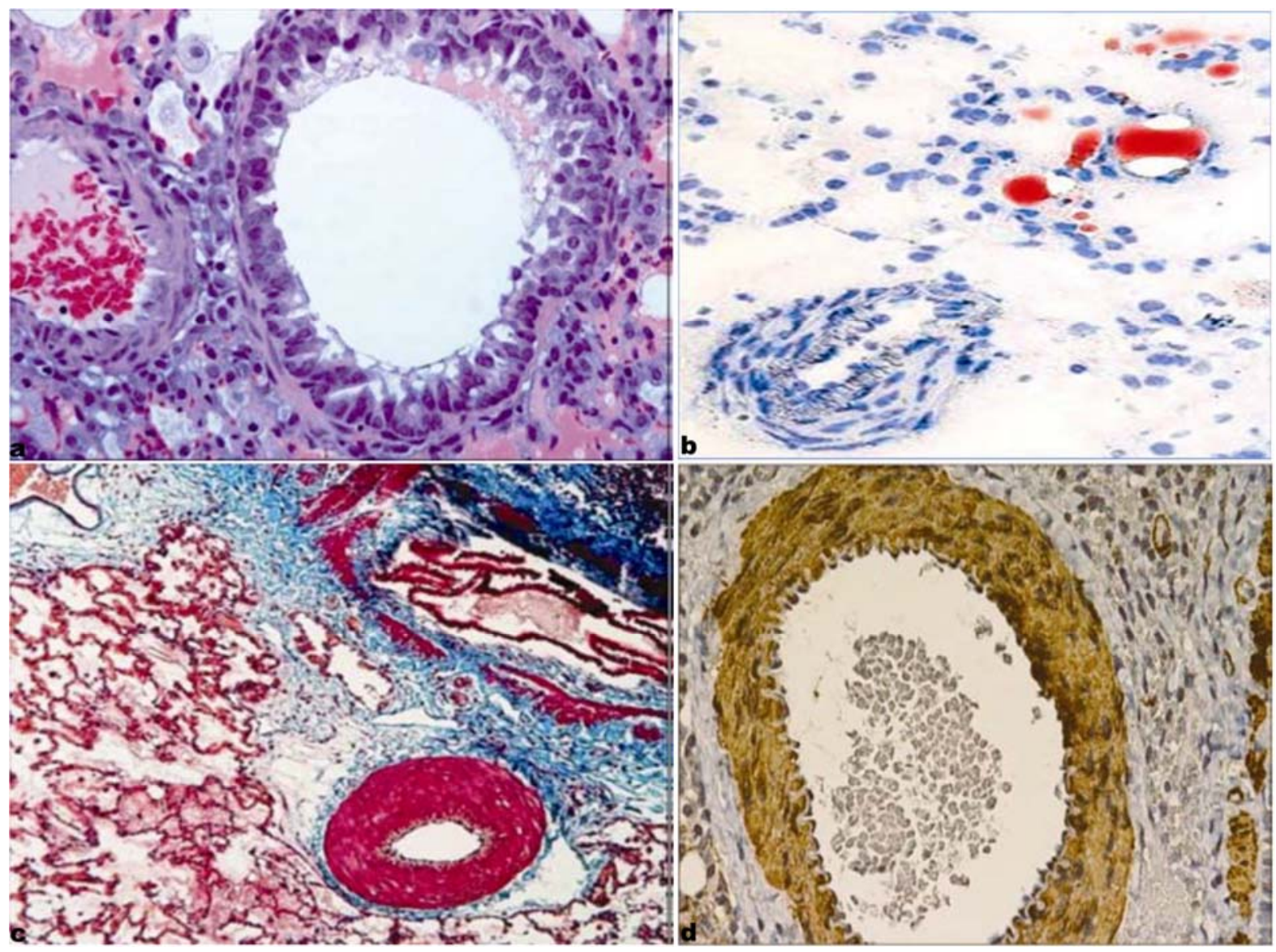

Figure 2. Lung of a triolein-treated rat at 48 hours. (a) H\&E staining shows the thickening of the vessels with decreased lumen patency, and inflammatory cells are present $(\times 400)$; (b) Oil Red O staining shows fat droplets in the alveolar spaces and in the small caliber vessels ( $\times 400)$; (c) Trichrome stain shows fibrosis in the vessels and in the septa ( $\times 100)$; (d) SMA stain also shows fibrosis in the same vessels $(\times 400)$. 
When the rats were allowed to survive up to 11 days, they clinically recovered with only modest pulmonary inflammation persisting and right ventricle dilatation returning within normal range. However a slow but progressive inflammatory process mostly affecting the small caliber pulmonary arteries and the septa was evident at three and six weeks despite a weight gain and behavior similar to that of the controls ${ }^{[10]}$. Fat droplets persisted in their lungs. When a "second hit" by LPS i.p. administration was given to the triolein treated rats, pulmonary vaso-constriction and inflammation returned to be very severe, similar, in their morphology to that observed in the acute period (48-96) hours post triolein ${ }^{[11]}$. Fat droplets were still present in the lungs ${ }^{[11]}$.

\section{Discussion}

Although the first case of FES was described in a patient with a fractured femur as early as 1873, the criteria for the syndrome's clinical definition were proposed by Gurd and Wilson only in $1974{ }^{[1]}$. Most of the attention was given, at that time, to the fractures of the long bones ${ }^{[13]}$, and only more recently FES cases were also described as one of the consequences of a variety of other surgical procedures such as percutaneous vertebroplasty, liposuction, or different surgical interventions in obese patients ${ }^{[14-16]}$.

Hypoxemia and tachycardia are observed in about $90 \%$ of the cases, reflecting an obstruction of the pulmonary circulation ${ }^{[14]}$; In a sheep model of vertebroplasty where cement was injected in the animal's spine, fat emboli were histologically demonstrated in the lungs of the treated animals ${ }^{[17,18]}$. In humans, the release of the fat particles triggers a localized pulmonary inflammatory reaction clinically identifiable with acute respiratory distress syndrome (ARDS) ${ }^{[19]}$. The same histopathological pulmonary damage is what we observed in our rat model, thus underlining the significant similarities to the conditions observed in humans ${ }^{[9,10]}$. The emboli observed in the lumen of small caliber pulmonary arteries and later on in the organ structure, especially in the subpleural spaces, are likely to generate a release of angiotensin 2 from various components of the organ such as the endothelial cells, the macrophages, mast cells and fibroblasts, and in our fat embolism model, we were able to prevent pulmonary vasoconstriction and the dilatation of the right ventricle of the heart both by administration of the angiotensin 1 converting enzyme inhibitor, captopril or the angiotensin 2 type- 1 receptor blocker, losartan when the drugs were given one hour after the triolein injection ${ }^{[20]}$. More recently, we also observed in the same rodent model a diffuse presence of macrophages positively stained for renin, thus adding more evidence of the role played by RAS in the pathogenesis of the condition ${ }^{[21]}$. The case of FES following percutaneous vertebroplasty reported by Ahmadzai and his collaborators had a previous history of systemic lupus erythematosus and vasculitis ${ }^{[16]}$. Diabetes, renal damage in addition to severe obesity and pre-eclampsia were present in our patient. These clinical conditions, the vasculopathy in particular, may be a predisposing factor in the FES pathogenesis.

Other predisposing factors could be the extensive surgical procedures the patient underwent in 2012, on the spine and the long bones. If some damage were done to the lungs during those procedures which was not observed or reported, the condition may resemble what we observed in our "second hit" experiment where LPS was given six weeks after triolein treatment ${ }^{[11]}$. The administration of angiotensin receptor blocker, losartan administration protected the rats from the pulmonary damage, especially the vasculitis and the fibrosis in this chronic model ${ }^{[22]}$. All these experimental observations underline the multiplicity and complexity of the factors involved in the pathogenesis of FES and the requirement of accurate clinical evaluations of the patients before any surgical procedure potentially leading to FES. It also underlines the necessity of a long term follow up of the patients post operatively.

\section{Acknowledgements}

The histopathology laboratory of the Department of pathology, Truman Medical Center, and Mr. Joe Moran for his assistance for the organization of the photographic material. Support was received by the Mary Catherine Geldmacher Research Foundation, Saint Louis, MO. 


\section{References}

[1] Gurd AR, Wilson RI. The fat embolism Syndrome. J Bone Joint Surg Br. 1974; 56B: 408-16. PMid: 4547466.

[2] Talbot M, Schemitsch EH. Fat embolism Syndrome: history, definition, and epidemiology. Injury. 2006: 37(suppl 4); S 3-7. PMid: 16990059. http://dx.doi.org/10.1016/j.injury.2006.08.035

[3] Syed MI, Jan S, Patel NA, et al. Fatal fat embolism after vertebroplasty: identification of the high -risk patient. AJNR Am J Neuroradiol. 2006; 27: 343-5. PMid: 16484407.

[4] Herridge MS, Tansey CM, Matté A, et al. Functional disability 5 years after acute respiratory distress syndrome. N Engl J Med. 2011; 364: 1293-304. PMid: 21470008. http://dx.doi.org/10.1056/NEJMoa1011802

[5] Fan E, Dowdy DW, Colantuoni E, et al. Physical complications in acute lung injury survivors: a two-year longitudinal prospective study. Crit Care Med. 2014; 42: 849-59. PMid: 24247473. http://dx.doi.org/10.1097/CCM.0000000000000040

[6] King EG, Wagner WW, Jr Ashbaugh DG, et al. Alterations in pulmonary microanatomy after fat embolism. In vivo observations via thoracic window of the oleic acid- embolized canine lung. Chest. 1971; 59: 526-30. PMid: 4952551. http://dx.doi.org/10.1378/chest.59.5.524

[7] Lui DD, Hsieh NK, Chen HI. Histopathological and biochemical changes following fat embolism with administration of corn oil micelles: a new animal model for fat embolism syndrome. J Bone Joint Surg Br. 2008; 90: 1517-21. PMid: 18978276.

[8] Jacobvitz-Derks D, Derks CM. Pulmonary neutral fat embolism in dogs. Am J Pathol. 1979; 95: 29-42. PMid: 434112.

[9] McIff TE, Poisner AM, Herndon B, et al. Fat embolism: evolution of histopathological changes in the rat lung. J Orthop Res. 2010; 28: 191-7. PMid: 19688870. http://dx.doi.org/10.1002/jor.20963

[10] Poisner AM, Adler F, Uhal B, et al. Persistent and progressive pulmonary fibrotic changes in a model of fat embolism. J Trauma Acute Care Surg. 2012; 72: 992-8. PMid: 22491616. http://dx.doi.org/10.1097/TA.0b013e31823c96b0

[11] Molteni A, Torres-Romero L, Black E, et al. Fat embolism syndrome following bone fracture may be exaggerated by LPS. Oral presentation. Proceedings of meeting online on Experimental Biology; 2013 May 20-24; Boston, United States. Abstract 3641.

[12] Kesh S, Molteni A, Stevens TM, et al. The pulmonary vessel adventitia: role in the pathology of fat embolism. Proceedings of the meeting online on Experimental Biology. 2014 April; 26-30, San Diego, California; 2014: Abstract LB 511.

[13] Glazer JL, Onion DK. Fat embolism syndrome in a surgical patient. J Am Board Fam Pract. 2001; 14: 310-3. PMid: 11458973.

[14] Childers JC Jr. Cardiovascular collapse and death during vertebroplasty. Radiology. 2003; 228: 902. PMid: 12954907. http://dx.doi.org/10.1148/radiol.2283030235

[15] Chen HL, Wong CS, Ho ST, et al. A lethal pulmonary embolism during percutaneous vertebroplasty. Anesth Analg. 2002; 95: 1060-2. PMid: 12351294.

[16] Ahmadzai H, Campbell S, Archis C, et al. Fat embolism syndrome following percutaneous vertebroplasty: a case report. Spine J. 2014; 14: e1-5. PMid: 24314905. http://dx.doi.org/10.1016/j.spinee.2013.09.021

[17] Aebli N, Krebs J, Davis G, et al. Fat embolism and acute hypotension during vertebroplasty: an experimental study in sheep. Spine. 2002; 27: 460-6. PMid: 11880830. http://dx.doi.org/10.1097/00007632-200203010-00005

[18] Krebs J, Ferguson SJ, Hoerstrup SP, et al. Influence of bone marrow fat embolism on coagulation activation in an ovine model of vertebroplasty. J Bone Joint Surg Am. 2008; 90: 349-56. http://dx.doi.org/10.2106/JBJS.G.00058

[19] Porpodis K, Karanikas M, Zarogoulidis P, et al. Fat embolism due to bilateral femoral fracture: a case report. Int J Gen Med. 2012; 5: 59-63. PMid: 22287848. http://dx.doi.org/10.2147/IJGM.S28455

[20] McIff TE, Poisner AM, Herndon B, et al. Mitigating effects of captopril and losartan on lung histopathology in a rat model of fat embolism. J Trauma. 2011; 70: 1186-91. PMid: 20693918. http://dx.doi.org/10.1097/TA.0b013e3181e50df6

[21] Poisner A, Herndon B, Al Hariri A, et al. Renin as a mediator of pulmonary damage caused by fat embolism and LPS. Proceedings of the meeting online on Experimental Biology; 2013 May 20; Boston, United States; 2013: Abstract 10471.

[22] Poisner A, Herndon B, Bass D, et al. Losartan protects the lung from chronic cellular damage induced by fat embolism. Proceedings of the meeting online on Experimental biology; 2014 April 26-30; San Diego, California: Abstract LB 512. 